\title{
Mechanical, Thermal and Electrical Properties of Lotus-Type Porous Metals
}

\author{
Hideo Nakajima \\ The Wakasa Wan Energy Research Center, Tsuruga, Fukui, Japan \\ Email: hnakajima@werc.or.jp
}

How to cite this paper: Nakajima, $H$. (2018) Mechanical, Thermal and Electrical Properties of Lotus-Type Porous Metals. Materials Sciences and Applications, 9, 258-267.

https://doi.org/10.4236/msa.2018.92017

Received: November 23, 2017

Accepted: February 4, 2018

Published: February 7, 2018

Copyright $\odot 2018$ by author and Scientific Research Publishing Inc. This work is licensed under the Creative Commons Attribution International License (CC BY 4.0).

http://creativecommons.org/licenses/by/4.0/

\begin{abstract}
Lotus-type porous metals are fabricated by directional solidification of molten metals dissolving hydrogen. Unidirectional pores cause various anisotropic features of mechanical, thermal and electrical properties. Tensile, compressive and fatigue strength parallel to the pore direction are higher than those perpendicular to the pore direction. Besides, thermal and electrical conductivities parallel to the pore direction are higher than those perpendicular to the pore direction. Such anisotropy is attributed to the difference in the pinning cross-sectional area due to the dislocations and the scattering cross-sectional area due to electrons. The pore cross-sectional area perpendicular to the pore direction is much larger than that parallel to the pore direction. It is surmised that such difference in pore cross-section results in the anisotropy in mechanical, thermal and electrical properties.
\end{abstract}

\section{Keywords}

Lotus Metals, Porous Metals, Mechanical Properties, Thermal Conductivity, Electrical Conductivity, Solidification, Porosity, Hydrogen

\section{Introduction}

Porous and foamed metals exhibit various characteristics such as low density and large surface area, which differ from bulk metals. These metals are expected to be used as lightweight materials, catalyst carrier, electrodes, vibration and acoustic energy damping materials, impact energy absorption materials, etc. [1] [2]. Most of the porous metals possess isotropic porous morphology. Among them, lotus-type and gasar-processed porous metals have attracted much attention because those possess long cylindrical pores aligned in one direction, which are anisotropic porous morphology. (Hereafter we call lotus metals.) While various mechanical and physical properties of isotropic porous metals usually exhi- 
bit isotropic characteristic, these properties of lotus metals show anisotropic features, depending upon the pore direction which are peculiar to lotus metals. It is considered that such anisotropy is resulted from the difference in the pinning cross-sectional area due to the dislocations and the scattering cross-sectional area due to electrons. The review describes anisotropic features of mechanical, thermal and electrical properties in order to elucidate the anisotropic mechanism.

\section{Fabrication of Lotus Metals}

Lotus metals are fabricated through unidirectional solidification of the melt in hydrogen atmosphere. When the melt dissolving hydrogen is solidified, insoluble hydrogen in the solid precipitates and evolves directional long pores. To fabricate lotus metals, two kinds of techniques are necessary: solidification techniques and hydrogen-supplying methods. The melt-routed solidification techniques are adopted: mold casting technique [3] [4], continuous zone technique [5], and continuous casting technique [6]. Among them, the most superior technique is the continuous casting technique, which is shown in Figure 1. This technique can control the solidification velocity and produce long sized ingots of lotus metals, which is suitable for mass-production.

Since the difference in gas solubility between liquid and solid is utilized to fabricate lotus metals, gas such as hydrogen, nitrogen or oxygen dissolved in the melt is indispensable. In order to dissolve the gas into the melt, two methods are available. In high-pressure gas method (PGM), the fabrication chamber is filled with the gas, which is dissolved up to the equilibrium gas concentration into the molten metal under a given gas pressure [2]. On the other hand, in thermal decomposition method (TDM), gas compounds such as hydride and nitride are

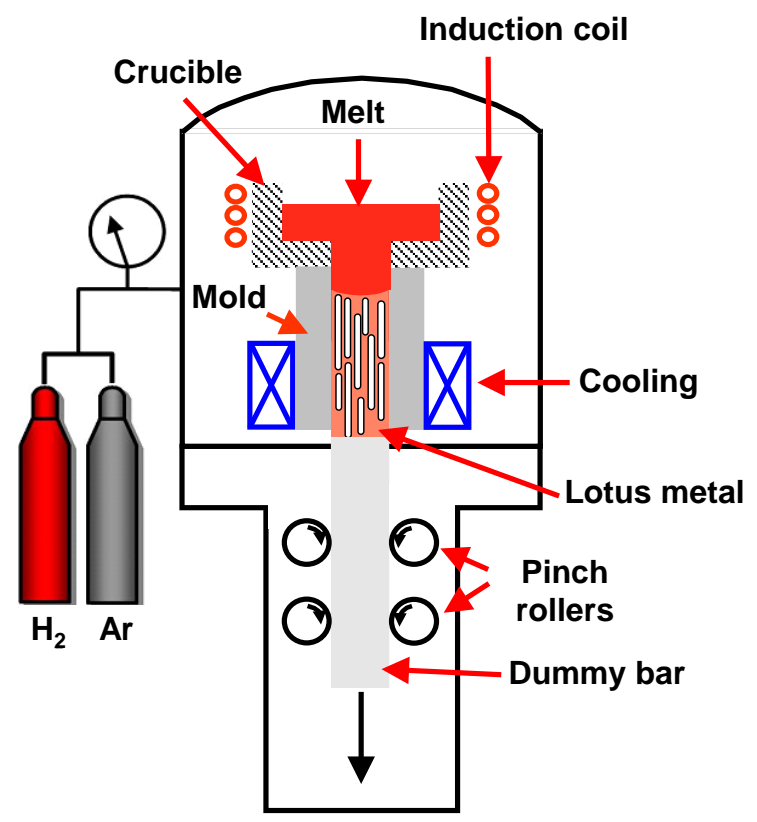

Figure 1. Schematic drawing of continuous casting apparatus. 
used as dissolving gas sources instead of high-pressure gas, so that a high-pressure chamber is not necessary [7]. After the gas compounds is added into the melt, the compounds simultaneously react with the molten metal to dissolve the gas. Usually argon or vacuum is used as an atmosphere during melting and solidification in order to prevent oxidation and impurity inclusion. Comparing both methods, PGM requires high-pressure gas and high-pressure chamber, while TDM does not require high-pressure gas and chamber, but inclusion of metallic constituent elements inherent from the gas compounds is inevitable in lotus metals.

\section{Mechanical Properties of Lotus Metals}

\subsection{Tensile Strength}

The ultimate tensile strength of specimens with cylindrical pore direction parallel to the tensile direction is plotted against the porosity in Figure 2 [8]. The data points for the ultimate tensile strength lie on a straight line which passes through the point of $0 \mathrm{MPa}$ at the porosity of $100 \%$; the specific ultimate tensile strength does not change by the pore existence. This suggests that the pores whose axes are aligned parallel to the tensile direction cause little stress concentration. Simple rule of mixture of the empty pores and the solid body can be applied to these specimens. The ultimate tensile strength of the specimens with cylindrical pores perpendicular to the tensile direction is also plotted against the porosity in Figure 2. The strength is much lower than that of specimens with the pores parallel to the tensile direction. Such decrease in the ultimate tensile strength is suggested to be attributed to the stress concentration. The stress concentration occurs at a location in an object where stress is concentrated. Over the area where

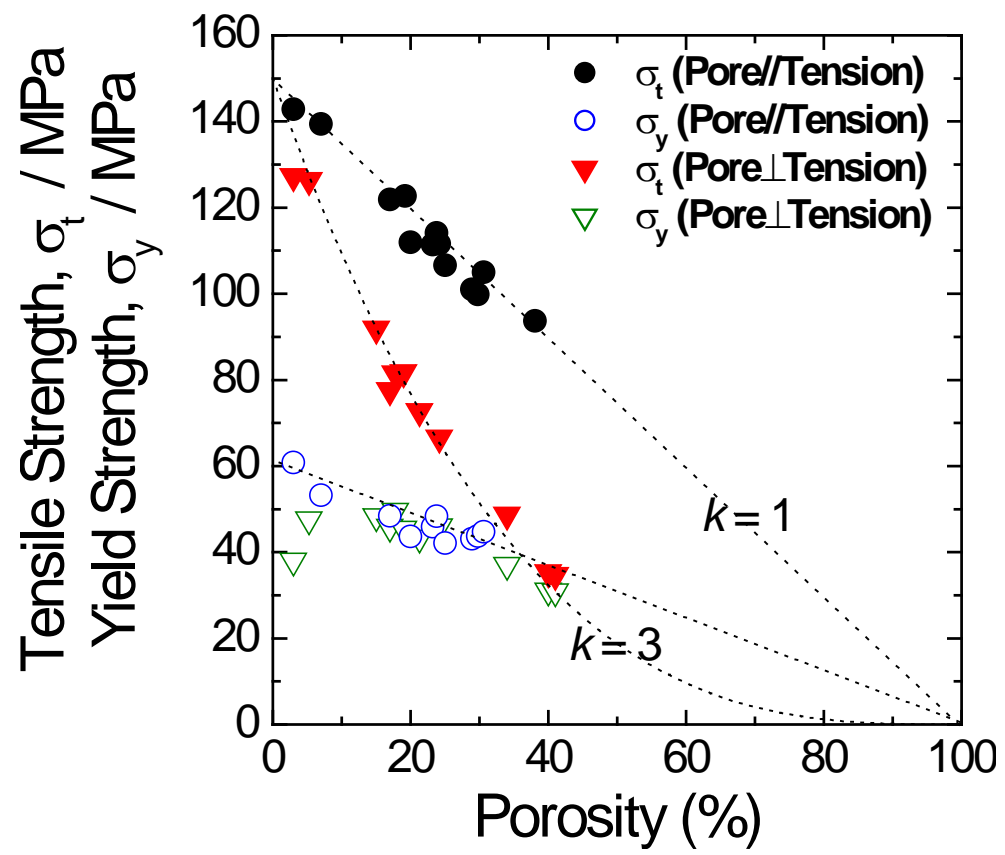

Figure 2. Porosity dependence of ultimate tensile strength of lotus copper. 
force is evenly distributed, the stress is uniform. However, if the pores exist, the stress is accumulated around the pores so that the stress concentration takes place. According to the load-bearing model and stress concentration model, the tensile strength $\sigma$ can be expressed by

$$
\sigma=\sigma_{0}(1-p)^{K}
$$

where $p$ is the porosity, $\sigma_{0}$ is the tensile strength of non-porous metal and $K$ is a constant which is called as the stress concentration coefficient. For the specimen with the cylindrical pores aligned parallel to the tensile direction, the value of $K$ approaches one, where no stress concentration takes place. For the specimen with cylindrical pores oriented perpendicular to the tensile direction, the value of $K$ approaches 3, suggesting the stress concentration takes place. Those experimental data are fitted well to the Equation (1). Therefore, it is suggested that (a) the ultimate tensile strength for lotus metal with cylindrical pores parallel to the tensile direction exhibits no stress concentration and (b) the ultimate tensile strength for lotus metal with cylindrical pores perpendicular to the tensile direction shows stress concentration.

\subsection{Compressive Strength}

Figure 3 shows the porosity dependence of the yield stress of lotus stainless steel [9]. The yield stress of lotus metal with cylindrical pores parallel to the compressive direction, $\sigma_{y \|}$ decreases almost linearly with increasing porosity, while the yield stress of lotus metal with cylindrical pores perpendicular to the compressive direction, $\sigma_{y \perp}$ rapidly decreases. Thus, $\sigma_{y \perp}$ is lower than $\sigma_{y \|}$ over the whole range of porosity. The yield stress of lotus stainless steel is also expressed by the power-law Equation (1). The values of $K$ were found to be 1.0 and 2.4 for the direction parallel and perpendicular to the pore direction, respectively.

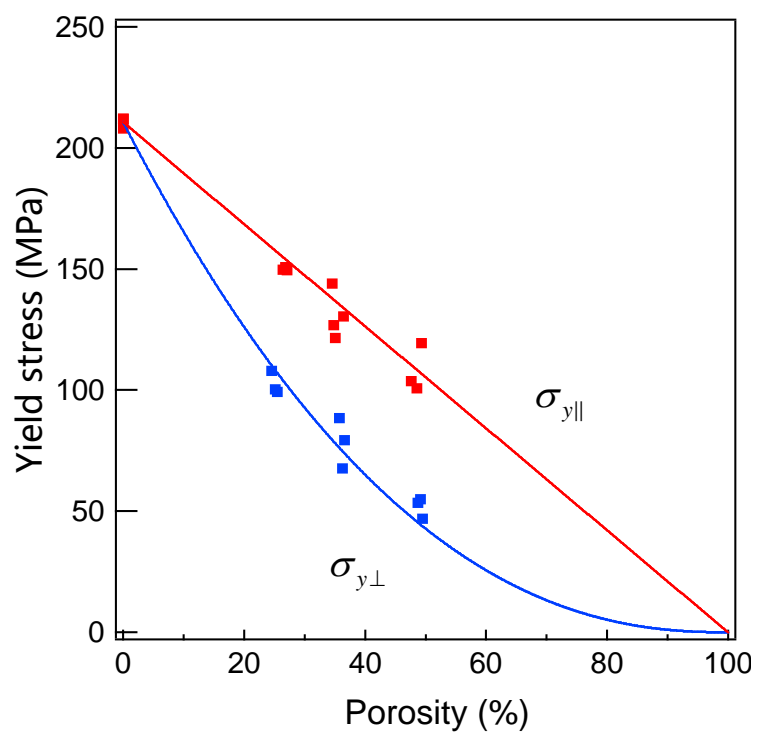

Figure 3. Porosity dependence of yield compressive stress of lotus stainless steel. 


\subsection{Fatigue Strength}

Figure 4 shows the log plots of the fatigue strength $\sigma_{a}$ against the number of cycles to failure $N_{f}$ for non-porous and lotus copper, where cyclic stress was applied in the direction (a) parallel and (b) perpendicular to the longitudinal axis of pores [10]. The lines denote the following function fitted to the experimental data:

$$
\log \sigma_{a}=C \log N_{f}+D
$$

where $C$ and $D$ are fitting coefficients. For both directions the stress amplitude decreases with increasing numbers of cycles to failure of lotus copper and non-porous copper. Non-porous copper does not show anisotropy in the fatigue strength at finite life. On the other hand, the fatigue strength at finite life of lotus copper shows significant anisotropy; the fatigue strength in the perpendicular direction is lower than that in the parallel direction.

Figure 5 shows the porosity dependence of fatigue strength at $N_{f}=10^{5}$ of lotus copper. The fatigue strength $\sigma_{a}$ can be expressed by the Equation (1), where $K$ values for fatigue strength parallel and perpendicular to the pore direction are 0.9 and 2.7, respectively. The fatigue strength parallel to the pore direction decreases linearly with increasing porosity, where no stress concentration occurs. On the other hand, the fatigue strength perpendicular to the pore direction decreases rapidly with increasing porosity, which is attributed to the weakness due to stress concentration.

\section{Thermal and Electrical Properties of Lotus Metals}

\subsection{Thermal Conductivity of Lotus Copper}

The effective thermal conductivity of lotus copper $k_{\text {eff }}$ is defined by

$$
q=Q / A=-k_{e f f} \nabla T
$$

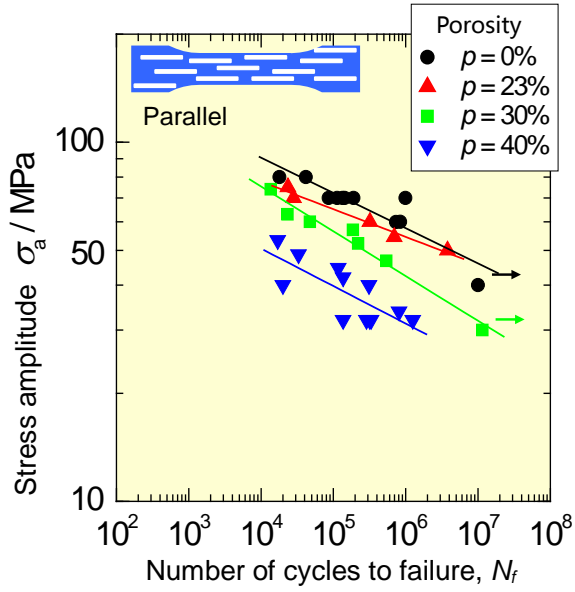

(a)

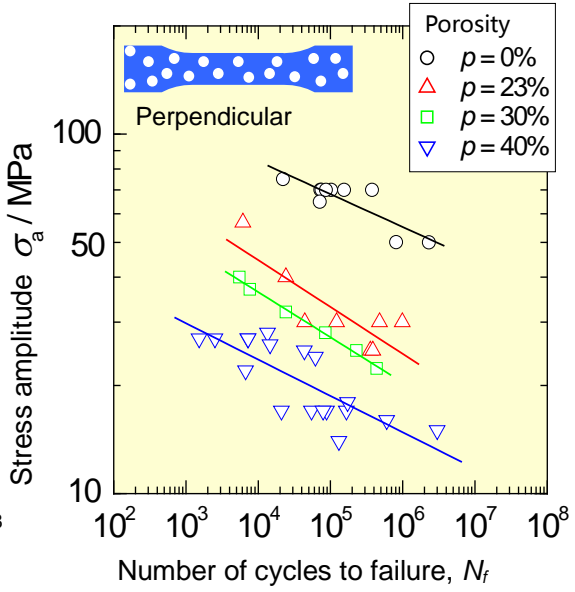

(b)

Figure 4. Log plots of stress amplitude against the number of cycles to failure for lotus copper, where cyclic stress was applied in the direction (a) parallel and (b) perpendicular to the longitudinal axis of cylindrical pores. 
where $q$ is the heat flux from heat flow $Q$ divided by heat flowing through the cross-sectional area $A$ in lotus copper including pores, and $T$ is the temperature in lotus copper. The effective thermal conductivity of lotus copper is anisotropic. The parallel and perpendicular effective thermal conductivities, $k_{\text {eff } \|}$ and $k_{\text {eff } \perp}$ of lotus copper are defined as the thermal conductivities for heat flow parallel and perpendicular to the pore axis, respectively.

Figure 6 presents the experimental apparatus for measuring the effective thermal conductivity. A cylindrical specimen with a diameter of $30 \mathrm{~mm}$ and a

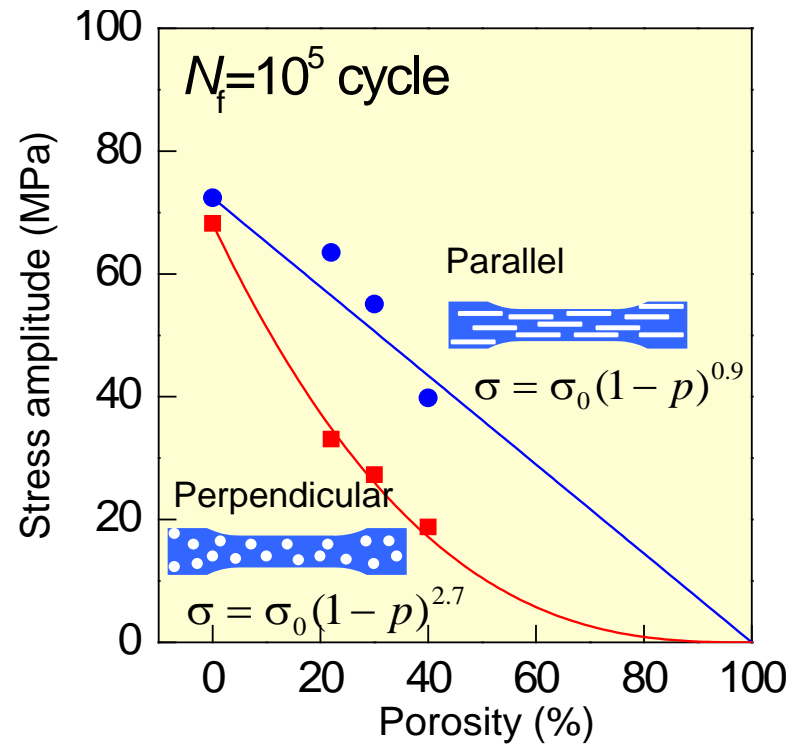

Figure 5. Porosity dependence of fatigue stress amplitude of lotus copper.

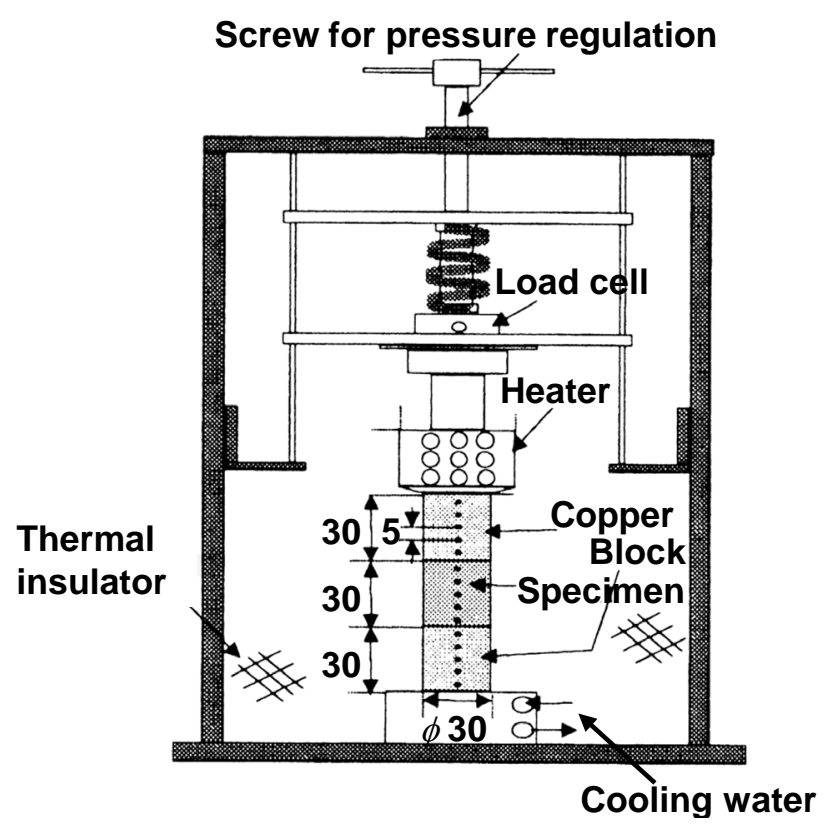

Figure 6. Experimental apparatus for measuring effective thermal conductivity. 
length of $30 \mathrm{~mm}$ was located between upper and lower copper rods of known thermal conductivity. The upper rod was heated by electrical heaters from the top surface, while the bottom surface of the lower rod was cooled by circulated water in order to transmit a certain amount of heat through the specimen. The temperatures were measured by K-type thermocouples.

Since the heat flow cross-sectional areas parallel to the pore axis in lotus copper is proportional to $(1-p)$, the effective thermal conductivity $k_{\text {eff } \|}$ is expressed by the following equation:

$$
k_{\text {eff } I \|} / k_{s}=1-p
$$

where $k_{s}$ is the thermal conductivity of non-porous copper. The effective thermal conductivity perpendicular to the pores can be expressed by

$$
k_{\text {eff } \perp} / k_{s}=(1-p) /(1+p)
$$

Figure 7 shows a comparison between the experimental data and the results evaluated by the analytical Equations (4) and (5) [11]. The experimental data are in good agreement with the results evaluated by the analytical equations. The effective thermal conductivity perpendicular to pores is lower than that parallel to pores. Such anisotropy is attributed to anisotropic scattering of the electrons with pores.

\subsection{Electrical Conductivity of Lotus Nickel}

Electrical conductivity of lotus nickel was measured by the four-probe method at room temperature. Figure 8 shows the porosity dependence of the electrical conductivity of lotus nickel, $\sigma_{\|}$and $\sigma_{\perp}$, where $\sigma_{\|}$and $\sigma_{\perp}$ are the electrical conductivity parallel and perpendicular to the pore direction [12]. For the electrical conductivity parallel to the pore direction, the specific electrical conductivity is almost constant. This is because the flow direction of the electrical current

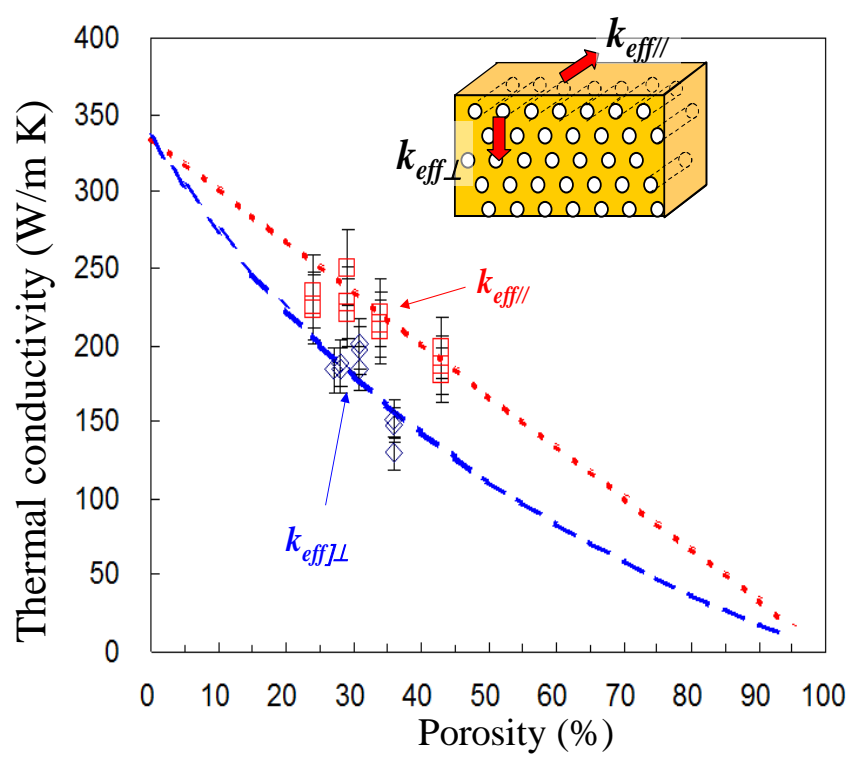

Figure 7. Porosity dependence of thermal conductivity of lotus copper. 


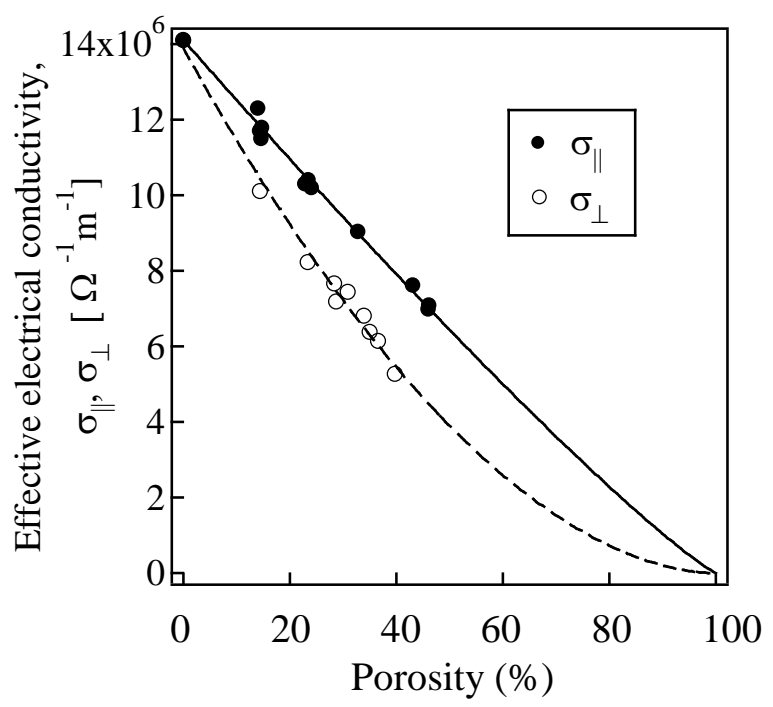

Figure 8. Porosity dependence of electrical conductivity of lotus nickel.

in the matrix is almost parallel to the applied electric field. For the perpendicular direction, the electrical conductivity decreases rapidly with increasing porosity. The electrical conductivity in the perpendicular direction is much lower than that in parallel direction. The electrons scatter with pores in lotus metals so that pores decrease conductivity. Since the pores are anisotropic in parallel and perpendicular directions, the scattering cross-sectional area is different in both directions. Thus, the electrical conductivity exhibits anisotropy.

\section{Anisotropic Behavior of Mechanical, Thermal and Electrical Properties}

The porosity dependence of mechanical, thermal and electrical properties is similarly fitted well to the power-law Equation (1). The mechanical properties result from dislocation behavior, while the thermal and electrical conductivity result from electron behavior. During deformation, the dislocations move in the crystal. If the pores exist, the dislocations are piled up around the pores and the stress concentration takes place, which affect the decrease in the strength perpendicular to the pores. Thus, the pores are considered as pinning sites impeding further dislocation propagation. On the other hand, thermal conduction is attributed to lattice conduction and electron conduction. In metals, electron conduction is dominant. In the lotus metals, the decrease in thermal conductivity occurs by electron scattering with the pores. Besides, the electrical conduction is responsible to the movement of current carriers (electrons). The decrease in the electrical conductivity in lotus metals is attributed to the scattering of electrons with pores. Thus, the pores are also considered as obstacles, that is, the scattering sites for electrons.

Figure 9 shows the cross-section of pores in lotus metals parallel and perpendicular to the pore direction. Cross-sectional area $S$ is evaluated approximately to be; 


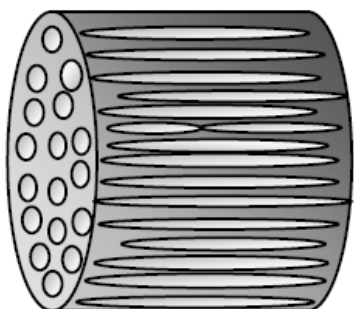

(a)

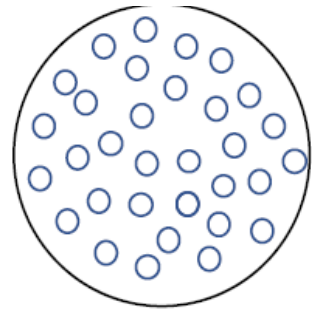

(b)

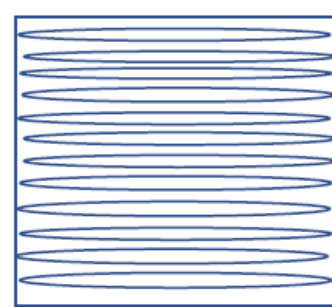

(c)

Figure 9. (a) Overview of lotus metal, (b) cross-section of the pores whose direction is parallel to solidification direction, and (c) cross-section of the pores whose direction is perpendicular to solidification direction.

$$
\begin{aligned}
& \text { Parallel to the pore direction } S_{\|}=\pi r^{2} n \\
& \text { Perpendicular to the pore direction } S_{\perp}=2 r l n
\end{aligned}
$$

where $n$ is the number of pores per unit volume, $r$ is the radius of pore and $l$ is the length of pore. Usually $l \gg r$ so that $S_{\perp}>S_{\|}$.

Therefore, the pinning cross-sectional or the scattering cross-sectional area perpendicular to the pore direction is much larger than that parallel to the pore direction. It is surmised that such difference causes more remarkable scattering difference and results in the anisotropy in mechanical, thermal and electrical properties.

\section{Summary}

The present review summarizes several peculiar features for lotus metals.

1) Unidirectional pores inherent from structure of lotus metals cause unique anisotropic characteristics of mechanical, thermal and electrical properties.

2) Tensile, compressive and fatigue strength parallel to the pore direction are higher than those perpendicular to the pore direction.

3) Thermal and electrical conductivities parallel to the pore direction are higher than those perpendicular to the pore direction.

4) Such anisotropy is attributed to the difference in the pinning cross-sectional area due to the dislocations and the scattering cross-sectional area due to the electrons.

Such anisotropic properties are expected to be utilized for application to various functional and structural materials.

\section{References}

[1] Banhart, J. (2001) Manufacture, Characterization and Application of Cellular Metals and Metal Foams. Progress in Materials Science, 46, 559-632. https://doi.org/10.1016/S0079-6425(00)00002-5

[2] Nakajima, H. (2007) Fabrication, Properties and Application of Porous Metals with Directional Pores. Progress in Materials Science, 52, 1091-1173. https://doi.org/10.1016/j.pmatsci.2006.09.001

[3] Shapovalov, V. (1994) Porous Metals. MRS Bulletin, XIX, 24-28. https://doi.org/10.1557/S0883769400039476 
[4] Nakajima, H., Hyun, S.K., Ohashi, K., Ota, K. and Murakami, K. (2001) Fabrication of Porous Copper by Unidirectional Solidification under Hydrogen and Its Properties. Colloids and Surface A: Physicochemical and Engineering Aspects, 179, 209-214. https://doi.org/10.1016/S0927-7757(00)00639-7

[5] Ikeda, T., Aoki, K. and Nakajima, H. (2005) Fabrication of Lotus-Type Porous Stainless Steel by Continuous Zone Melting Technique and Mechanical Property. Metallurgical and Materials Transactions A, 36, 77-86. https://doi.org/10.1007/s11661-005-0140-1

[6] Park, J.S., Hyun, S.K., Suzuki, S. and Nakajima, H. (2007) Effect of Transference Velocity and Hydrogen Pressure on Porosity and Pore Morphology of Lotus-Type Porous Copper Fabricated by Continuous Casting Technique. Acta Materialia, 55, 5646-5654. https://doi.org/10.1016/j.actamat.2007.06.022

[7] Nakajima, H. and Ide, T. (2008) Fabrication of Porous Copper with Directional Pores through Thermal Decomposition of Compounds. Metallurgical and Materials Transactions A, 39, 390-394. https://doi.org/10.1007/s11661-007-9402-4

[8] Hyun, S.K., Murakami, K. and Nakajima, H. (2001) Anisotropic Mechanical Properties of Porous Copper Fabricated by Unidirectional Solidification. Materials Science and Engineering, A299, 241-248.

https://doi.org/10.1016/S0921-5093(00)01402-7

[9] Ide, T., Tane, M., Ikeda, T., Hyun, S.K. and Nakajima, H. (2006) Compressive Properties of Lotus-Type Porous Stainless Steel. Journal of Materials Research, 21, 185-193. https://doi.org/10.1557/jmr.2006.0016

[10] Seki, H., Tane, M., Otsuka, M. and Nakajima, H. (2007) Effect of Pore Morphology on Fatigue Strength and Fracture Surface of Lotus-Type Porous Copper. Journal of Materials Research, 22, 1331-1338. https://doi.org/10.1557/jmr.2007.0164

[11] Ogushi, T., Chiba, H., Nakajima, H. and Ikeda, T. (2004) Measurement and Analysis of Effective Thermal Conductivities of Lotus-Type Porous Copper. Journal of Applied Physics, 95, 5843-5847. https://doi.org/10.1063/1.1691188

[12] Tane, M., Hyun, S.K. and Nakajima, H. (2005) Anisotropic Electrical Conductivity of Lotus-Type Porous Nickel. Journal of Applied Physics, 97, Article ID: 103701. https://doi.org/10.1063/1.1897069 